\title{
FUBP1 mediates the growth and metastasis through TGFß/Smad signaling in pancreatic adenocarcinoma
}

\author{
YUE ZHANG $^{1,2^{*}}$, JINLIAN CHEN $^{1 *}$, NVSHI ZHOU $^{2}$, YUN LU $^{2}$, JINGWEN LU $^{3}$, \\ XIN XING ${ }^{4}$, HUA CHEN $^{1}$ and XINGXING ZHANG ${ }^{1}$ \\ ${ }^{1}$ Department of Gastroenterology, Anhui University of Science and Technology Affiliated Fengxian Hospital, \\ Shanghai University of Medicine and Health Sciences Affiliated Sixth People's Hospital South Campus, \\ Shanghai Fengxian District Central Hospital, Shanghai 201499; \\ ${ }^{2}$ School of Medicine, Anhui University of Science and Technology, Huainan, Anhui 232001; \\ ${ }^{3}$ Department of Pathology, Anhui University of Science and Technology Affiliated Fengxian Hospital; \\ ${ }^{4}$ Central Laboratory, Anhui University of Science and Technology Affiliated Fengxian Hospital, \\ Shanghai University of Medicine and Health Sciences Affiliated Sixth People's Hospital South Campus, \\ Shanghai Fengxian District Central Hospital, Shanghai 201499, P.R. China
}

Received November 11, 2020; Accepted January 29, 2021

DOI: $10.3892 / \mathrm{ijmm} .2021 .4899$

\begin{abstract}
Recent studies have reported that the expression levels of far upstream element-binding protein 1 (FUBP1) were upregulated and served a crucial role in several types of cancer. However, the underlying molecular mechanisms and clinical significance of FUBP1 in pancreatic adenocarcinoma (PAAD) remain unclear. The present study aimed to determine the expression levels of FUBP1 in patients with PAAD and subsequently investigated the biological functions and mechanisms of FUBP1 using in vitro assays. FUBP1 expression levels and survival outcomes in patients with PAAD were analyzed using The Cancer Genome Atlas and starBase databases. Reverse transcription-quantitative PCR was used to analyze the mRNA expression levels of FUBP1 in PAAD and adjacent normal tissues. In addition, the expres-
\end{abstract}

Correspondence to: Dr Xingxing Zhang or Dr Hua Chen, Department of Gastroenterology, Anhui University of Science and Technology Affiliated Fengxian Hospital, Shanghai University of Medicine and Health Sciences Affiliated Sixth People's Hospital South Campus, Shanghai Fengxian District Central Hospital, 6600 Nanfeng Road, Fengxian, Shanghai 201499, P.R. China

E-mail: simonzx1989@163.com

E-mail: chengjunyi999@126.com

*Contributed equally

Abbreviations: FUBP1, far upstream element-binding protein 1; PAAD, pancreatic adenocarcinoma; RT-qPCR, reverse-transcriptionquantitative PCR; EMT, epithelial-mesenchymal transition; DAB, diaminobenzidine; FBS, fetal bovine serum; DMEM, Dulbecco's modified Eagle's medium; CCK-8, Cell Counting Kit-8

Key words: pancreatic adenocarcinoma, far upstream element-binding protein 1, epithelial-mesenchymal transition, TGF $\beta /$ Smad pathway sion of FUBP1 was knocked down with small interfering RNA and overexpressed using FUBP1-overexpressed plasmids, and the effects on biological functions, including cell proliferation, migration and invasion, were investigated. Western blotting and immunofluorescence assays were used to determine the role of FUBP1 in epithelial-mesenchymal transition (EMT). The results of the present study revealed that the expression levels of FUBP1 were upregulated in PAAD tissues compared with adjacent normal tissues and the upregulated expression was significantly associated with poor survival. The knockdown of FUBP1 expression significantly inhibited the proliferative, migratory and invasive abilities of the PAAD PaTu8988 cell line, while the overexpression of FUBP1 promoted cell proliferation, migration and invasion in the PAAD SW1990 cell line. Furthermore, the knockdown of FUBP1 downregulated the expression levels of EMT-related markers, including $\mathrm{N}$-cadherin, $\beta$-catenin and vimentin, while the expression levels of E-cadherin were upregulated. The knockdown of FUBP1 was also revealed to regulate the TGF $\beta /$ Smad signaling cascade by downregulating phosphorylated-Smad2/3 and TGF $\beta 1$ expression levels. Conversely, the overexpression of FUBP1 reversed these effects. In conclusion, the findings of the present study indicated that FUBP1 may be a potential oncogene that mediates the EMT of PAAD via TGF $\beta /$ Smad signaling. These data suggested that FUBP1 may represent a potential biomarker for the diagnosis of PAAD or a target for the treatment of patients with PAAD.

\section{Introduction}

Pancreatic adenocarcinoma (PAAD) is one of the most lethal types of malignancy and has an extremely poor prognosis, with an overall 5-year survival rate of $<5 \%(1,2)$. The main reason for the poor prognosis is that it is difficult to diagnose PAAD at an early stage, because the cancer-specific symptoms usually only occur at an advanced stage (3). In addition, there are currently no effective treatments available for PAAD. 
Therefore, novel strategies to diagnose and prevent PAAD are required urgently.

Human far upstream element-binding protein 1 (FUBP1) was discovered to be an important regulator of transcription, mRNA splicing and translation by binding to far upstream element (FUSE), and is located in the reverse strand of chromosome 1p31.1 (4). Upon binding to FUSE, FUBP1 was reported to upregulate the expression levels of the oncogene Myc, which subsequently promoted cell growth and metastasis, and functioned as an oncogene by modulating the FUBP1/FUSE/Myc feedback loop (4-6). Notably, FUBP1 has also been reported to exert oncogenic roles in a variety of tumor types, including liver cancer $(7,8)$, glioma (9), neuroblastoma (10), renal cell carcinoma (11), lung cancer (12), tongue squamous cell carcinoma (13), esophageal squamous cell carcinoma (14), gastric cancer $(15,16)$ and colorectal cancer $(17)$. A previous study demonstrated that FUBP1 served as an oncogene and was associated with a poor prognosis in patients with PAAD (18). In addition, FUBP1 regulated the immune response by upregulating the expression levels of programmed death-ligand 1 in PAAD cells (18). However, the biological functions and molecular mechanisms of FUBP1 in PAAD remain unknown and require further investigations.

The present study analyzed the expression levels of FUBP1 in PAAD and adjacent normal tissues and determined the association between FUBP1 and clinical prognosis of PAAD. Moreover, the role of FUBP1 on the biological functions of PAAD cells, in addition to the potential mechanisms, were investigated in vitro.

\section{Materials and methods}

Patient studies. From September 2017 to December 2019, a total of 7 patients including 5 males and 2 females, with a mean age of 49.5 years (range, 40-66 years), diagnosed with PAAD at the Shanghai Fengxian District Central Hospital (Shanghai, China) were enrolled in the present study. All patients provided written informed consent prior to participation. The 7 pairs of PAAD and adjacent normal tissues (at least $3 \mathrm{~cm}$ away from the margin of PAAD tissues) were resected and collected following surgery. All the patients had not received radiotherapy or chemotherapy before the operation and were diagnosed with PAAD according to the pathological results. The patients with other malignant tumors were excluded. The study protocol was approved by the Ethics Committee of the Shanghai Fengxian District Central Hospital (approval no. 2017-Ethical Review-KY-05) and was performed in accordance with the principles of the Declaration of Helsinki.

Expression dataset. Gene expression RNAseq data of FUBP1 were downloaded from the UCSC Xena database (http://xena. ucsc.edu/) (19), which included PAAD samples [The Cancer Genome Atlas (TCGA), n=183] and normal samples [Genotype Tissue Expression (GTEx), $n=165]$. The correlation between gene expression and overall survival was downloaded from the starBase database (http://starbase.sysu.edu.cn/index.php) (20), which contained 176 PAAD patients.

Cell lines and culture. Human PAAD cell lines (BxPC-3, PaTu8988, PANC-1 and SW1990) were conserved at the
Central Laboratory of Shanghai Fengxian District Central Hospital, and were cultured in DMEM supplemented with 10\% FBS (both from Gibco; Thermo Fisher Scientific, Inc.), and maintained in a humidified incubator with $5 \% \mathrm{CO}_{2}$ at $37^{\circ} \mathrm{C}$.

Reverse transcription-quantitative PCR (RT-qPCR). Total RNA was extracted from tissues and cells using TRIzol ${ }^{\circledR}$ reagent (Invitrogen; Thermo Fisher Scientific, Inc.). Total RNA was reverse transcribed into cDNA using a PrimeScript ${ }^{\mathrm{TM}}$ RT Reagent kit (Takara Bio, Inc.) with the conditions of $15 \mathrm{~min}$ at $37^{\circ} \mathrm{C}$ and $5 \mathrm{sec}$ at $85^{\circ} \mathrm{C}$. qPCR was subsequently performed on an ABI 7300 Real-Time PCR system (Applied Biosystems; Thermo Fisher Scientific, Inc.) using a SYBR ${ }^{\circledR}$ Premix Dimmer Eraser kit (Takara Bio, Inc.). Thermocycling conditions for the qPCR were as follows: Initial denaturation at $95^{\circ} \mathrm{C}$ for $30 \mathrm{sec} ; 40$ cycles of denaturation at $95^{\circ} \mathrm{C}$ for $5 \mathrm{sec}$; annealing at $58^{\circ} \mathrm{C}$ for $30 \mathrm{sec}$, and extension at $72^{\circ} \mathrm{C}$ for $30 \mathrm{sec}$. The following primers pairs were used for the qPCR: FUBP1 forward, 5'-GGACAACACCCGAAAGGATA-3' and reverse, 5'-ATGTTCCAGTTGCCTTGACC-3'; and GAPDH forward, 5'-TTGGTATCGTGGAAGGACTCA-3' and reverse, 5'-TGT CATCATATTTGGCAGGTT-3' (all purchased from Sangon Biotech Co., Ltd.). The relative mRNA expression levels were quantified using the $2^{-\Delta \Delta \mathrm{Cq}}$ method (21). GAPDH was used as the internal control.

Cell transfection. Cells were transfected with small interfering RNA (siRNA/si) targeting FUBP1 (si-FUBP1), si-negative control (NC), FUBP1 overexpression (OE) vector (FUBP1-OE) or control vector using Lipofectamine ${ }^{\circledR} 2000$ reagent (Invitrogen; Thermo Fisher Scientific, Inc.) and Opti-MEM (Thermo Fisher Scientific, Inc.), according to the manufacturer's protocol. Briefly, PaTu8988 cells with a confluence of $50-60 \%$ in a 6 -well plate were transfected with 100 pmol si-FUBP1 or an equal volume of si-NC. For the transfection of plasmids, the cell density of SW1990 was $70-80 \%$ before transfection, with $5 \mu \mathrm{g}$ FUBP1-OE or VECTOR plasmids transfected in each well of a 6-well plate. Subsequently, the mixture of transfection reagents was replaced with $10 \%$ FBS-DMEM after $5 \mathrm{~h}$ at $37^{\circ} \mathrm{C}$. Then, the following assays were performed after post-transfected cells were incubated at $37^{\circ} \mathrm{C}$ for $48 \mathrm{~h}$. The sequences of the siRNAs (all from GenePharma, Co., Ltd.) were as follows: si-FUBP1 forward, 5'-GGUGCUGACAAACCUCUUATT-3' and reverse, 5'-UAAGAGGUUUGUCAGCACCTT-3'; si-NC forward, 5'-UUCUCCGAACGUGUCACGUTT-3' and reverse, 5'-ACGUGACACGUUCGGAGAATT-3'. The OE-FUBP1 plasmid contained full-length amplified sequences of FUBP1 cloned into a pCD513B plasmid (purchased from Biogot Biotechnology Co., Ltd).

Cell Counting Kit-8 (CCK-8) assay. Cell proliferation was analyzed using a CCK- 8 assay (Dojindo Molecular Technologies, Inc.), according to the manufacturer's protocol. Briefly, following transfection, cells were seeded into 96-well plates at a density of $5 \times 10^{3}$ cells/well and cultured for 10,24 , 48 or $72 \mathrm{~h}$. After the cells were washed twice with cold PBS, the mixture of $10 \mu \mathrm{l}$ CCK- 8 and $100 \mu 1$ serum-free DMEM was added into each well and the plates were incubated at $37^{\circ} \mathrm{C}$ 
for $45 \mathrm{~min}$. Subsequently, the absorbance was measured at a wavelength of $450 \mathrm{~nm}$ using a Wellscan MK3 microplate reader (Thermo Labsystems, Inc.).

Cell migration and invasion assays. Cell migration and invasion assays were performed in $8.0-\mu \mathrm{m}$ pore insert Transwell chambers (EMD Millipore) and Transwell invasion Matrigel chambers (Corning, Inc.), respectively. For the invasion assay, the Transwell membrane of the Matrigel chambers was precoated for $1 \mathrm{~h}$ at $37^{\circ} \mathrm{C}$ using serum-free DMEM. Briefly, DMEM supplemented with $10 \% \mathrm{FBS}$ was plated into the lower chambers and $5 \times 10^{4}$ cells in serum-free DMEM were plated into the upper chambers. Following incubation at $37^{\circ} \mathrm{C}$ for $24 \mathrm{~h}$, the non-migratory and non-invasive cells remaining in the upper chambers were gently removed with a cotton bud. The invasive and migratory cells in the lower chambers of the Transwell plates were stained with $0.1 \%$ crystal violet for $30 \mathrm{~min}$ at room temperature. The migratory and invasive cells were counted under a bright-field fluorescence microscope (Olympus Corporation; magnification, x200) in five randomly selected fields of view.

Wound healing assay. Upon transfected cells reaching 95\% confluence, an artificial wound was made in the cell monolayer by creating a scratch with a $200-\mu 1$ pipette tip. Since it was determined the pancreatic cells grow quickly, and, with the condition of 5\% FBS medium it was revealed that the scratch wound could not be measured at $48 \mathrm{~h}$. Thus, this assay was conducted by using $2 \%$ FBS medium to culture the cells (22). The cells were subsequently cultured in DMEM supplemented with $2 \% \mathrm{FBS}$ at $37^{\circ} \mathrm{C}$ after washing with PBS three times. Images of the wound were captured at 0,24 and $48 \mathrm{~h}$ using an OLYMPUS inverted microscope at a magnification of x100, and measured by the ImageJ software (version 1.52) (National Institutes of Health), respectively, to determine the cell migratory ability.

Western blotting. Protein was extracted from transfected cells using RIPA buffer (Beyotime Institute of Biotechnology), and protein concentration was determined using a BCA kit (Beyotime Institute of Biotechnology). Briefly, 40-60 $\mu \mathrm{g}$ equal amounts of protein were loaded per lane and separated by $10 \%$ SDS-PAGE, and transferred to PVDF membranes (EMD Millipore). The PVDF membranes were blocked using $5 \%$ non-fat milk in TBST for $1 \mathrm{~h}$ at $37^{\circ} \mathrm{C}$ and then incubated with primary antibodies at $4^{\circ} \mathrm{C}$ overnight. Following several washes using TBST, the PVDF membranes were incubated with specific secondary antibodies [(HRP-labeled goat anti-mouse IgG; cat. no. S0002; 1:5,000) or (HRP-labeled goat anti-rabbit IgG, cat. no. S0001; 1:5,000; both from from Affinity Biosciences)] for $2 \mathrm{~h}$ at room temperature. Finally, after washing the membranes three times with TBST, the protein bands were visualized using an ECL kit (EMD Millipore) and the bands were quantified using ImageJ software (version 1.52) (National Institutes of Health). The following antibodies were used: Anti-FUBP1 (68 kDa; product code ab192867, 1:1,000; Abcam), anti-E-cadherin (135 kDa; product no. 3195; 1:1,000), anti-N-cadherin (140 kDa; product no. 13116; 1:1,000), anti-vimentin ( $57 \mathrm{kDa}$; product no. $5741 ; 1: 1,000)$, anti- $\beta$-catenin (92 kDa; product no. 8480; 1:1,000), anti-Smad2/3 (52/60 kDa; product no. 3102; 1:1,000), anti-phosphorylated (p)-Smad2/3 (52/60 kDa; product no. 8828; 1:1,000), anti-TGF $\beta 1$ (12 kDa; product no. 3709; $1: 1,000)$, and anti- $\beta$-tubulin $(55 \mathrm{kDa}$; product no. 2128; 1:1,000; all from Cell Signaling Technology, Inc.) and anti- $\beta$-actin (42 kDa; cat. no. T0022, 1:5,000; Affinity Biosciences). $\beta$-actin and $\beta$-tubulin primary antibodies were used as the loading controls.

Immunofluorescence analysis. Following transfection with si-FUBP1 for $48 \mathrm{~h}$, cells were cultured on glass coverslips for $48 \mathrm{~h}$. The cells were subsequently fixed with $4 \%$ paraformaldehyde for $15 \mathrm{~min}$ at room temperature and blocked with $3 \%$ BSA (Beijing Solarbio Science \& Technology Co., Ltd.) for $1 \mathrm{~h}$ at room temperature. The cells were then incubated with the primary antibody overnight at $4^{\circ} \mathrm{C}$ prior to incubation with a Cy3-conjugated Goat Anti-Rabbit IgG secondary antibody (1:500; cat. no. A0516; Beyotime Institute of Biotechnology) in a dark room for $1 \mathrm{~h}$ at room temperature. The cell nuclei were counterstained with DAPI (Beyotime Institute of Biotechnology) in a dark room for $15 \mathrm{~min}$ at room temperature. Stained cells were visualized with a fluorescence microscope (Olympus Corporation; magnification, x200). The following primary antibodies were used: Anti-E-cadherin (cat. no. 3195; 1:200) and anti-vimentin (cat. no. 5741; 1:100; both from Cell Signaling Technology, Inc.).

Statistical analysis. Statistical analysis was performed using GraphPad Prism 5.0 software (GraphPad Software, Inc.). All data are presented as the mean \pm SD of $\geq 3$ independent experiments. Statistical differences were analyzed using a paired Student's t-test and one-way ANOVA followed by a Bonferroni's post hoc test. $\mathrm{P}<0.05$ was considered to indicate a statistically significant difference.

\section{Results}

FUBP1 expression levels are upregulated in PAAD and positively associated with clinical prognosis. The expression levels of FUBP1 in PAAD and normal tissues were analyzed using data from TCGA database (19). The results revealed that the expression levels of FUBP1 in PAAD tissues were significantly upregulated compared with normal pancreatic tissues (Fig. 1A). Thus, the clinical significance of FUBP1 in PAAD was determined using the starBase database (20). The long-term overall survival was determined to be associated with the expression levels of FUBP1 (Fig. 1B). Thus, the patients with PAAD with upregulated FUBP1 expression levels were at a higher risk of prognosis compared with patients with lower expression levels of FUBP1. Similarly, RT-qPCR analysis of seven pairs of PAAD and adjacent normal tissues revealed that FUBP1 mRNA expression levels were significantly upregulated in PAAD tissues compared with the corresponding adjacent normal tissues (Fig. 1C). These findings indicated that FUBP1 may serve as an oncogene and may be associated with a poor prognosis in patients with PAAD.

FUBP1 promotes $P A A D$ cell proliferation, migration and invasion. In human PAAD cell lines, PaTu8988, SW1990, BxPC-3 and Panc-1, the mRNA and protein expression levels of FUBP1 were analyzed using RT-qPCR and western blot- 
A

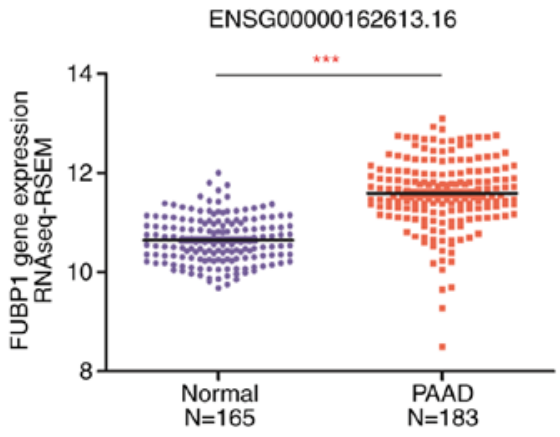

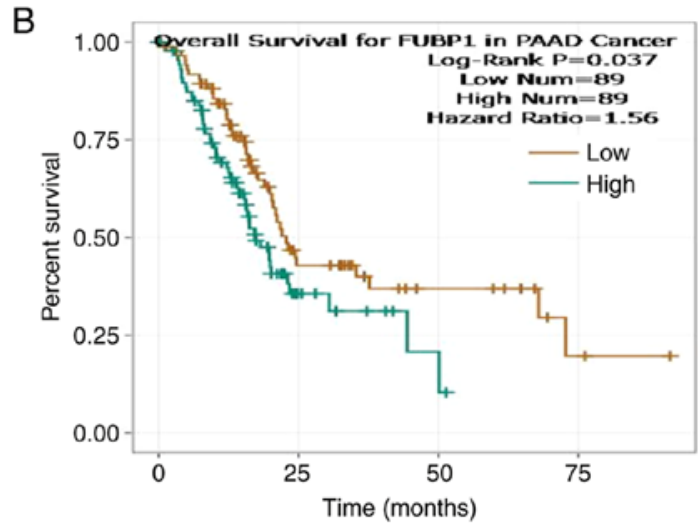

B

$\square$ Adjacent normal tissue $\infty$ PAAD tissue

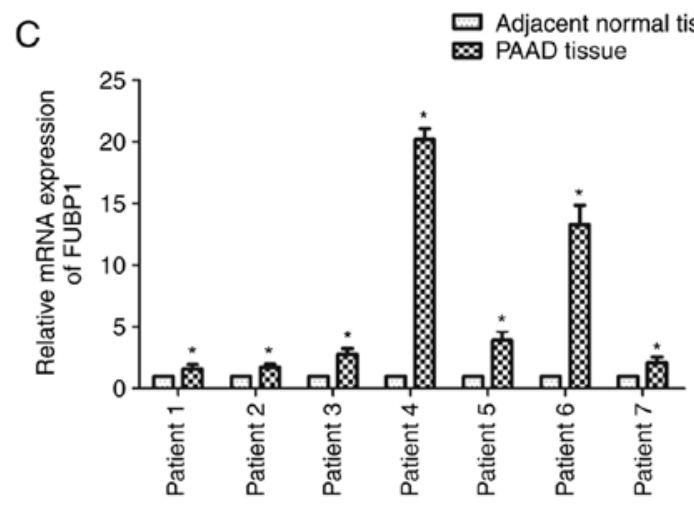

Figure 1. Upregulated FUBP1 expression levels are associated with a poor prognosis in patients with PAAD. (A) FUBP1 expression levels in PAAD and adjacent normal tissues from The Cancer Genome Atlas database. (B) starBase database was used to determine that, compared with patients with PAAD with low expression levels of FUBP1, patients with high expression levels of FUBP1 had a poorer overall survival ( $\mathrm{P}=0.037)$. (C) mRNA expression levels of FUBP1 were upregulated in seven human PAAD tissues compared with adjacent normal tissues. Data are presented as the mean \pm SD from three independent experiments. ${ }^{*} \mathrm{P}<0.05$ and ${ }^{* * * *} \mathrm{P}<0.01$. FUBP1, far upstream element-binding protein 1; PAAD, pancreatic adenocarcinoma.

ting. Among the four human PAAD cell lines, the highest expression levels of FUBP1 were observed in PaTu8988 cells, whereas the lowest expression levels were recorded in SW1990 cells (Fig. 2A). Thus, PaTu8988 cells were selected to further investigate the effects of the knockdown of FUBP1 expression in PAAD, while SW1990 cells were selected to determine the effects of the overexpression of FUBP1. The transfection efficiencies of cells transfected with si-FUBP1 or FUBP1-OE were determined using RT-qPCR and western blotting (Fig. 2B and C).

To investigate whether FUBP1 was associated with biological functions in PAAD, a CCK-8 assay was used to determine the effect of FUBP1 on cell proliferation, while wound healing and Transwell assays were conducted to determine the role of FUBP1 on cell migration and invasion, respectively. The data demonstrated that the knockdown FUBP1 expression significantly inhibited the proliferative ability of PaTu8988 cells (Fig. 3A), whereas the overexpression of FUBP1 promoted proliferation in SW1990 cells (Fig. 3B). Following the knockdown of FUBP1 expression levels in PaTu8988 cells, the results of the Transwell assays revealed that the number of migratory and invasive cells was significantly decreased (Fig. 4A, C and D). Notably, following the upregulation of FUBP1, the number of migratory and invasive cells was significantly increased in SW1990 cells (Fig. 4B, E and F). Similarly, the results of the wound healing assay also illustrated that the knockdown of FUBP1 expression inhibited the migratory ability of PaTu8988 cells (Fig. 4G and I). By contrast, the overexpression of FUBP1 increased the migratory ability of SW1990 cells (Fig. 4H and J). These data suggested that FUBP1 may promote the proliferation, migration and invasion of PAAD cells, which implied that FUBP1 may facilitate the adhesion of tumor cells to the extracellular matrix.

FUBP1 activates epithelial-mesenchymal transition (EMT) via the TGF $/$ Smad signaling pathway in PAAD cells. During tumor development, tumor cells constantly communicate with the surrounding microenvironment, which guides tumor cells undergoing EMT $(23,24)$. EMT is regarded as a pivotal step for promoting tumor invasion and metastasis and has been discovered to serve a role in the progression of PAAD (22). Since FUBP1 was revealed to promote PAAD cell migration and invasion, it was hypothesized that FUBP1 may be involved in EMT and influence cancer metastasis. To further determine the effect of FUBP1 on the progression of PAAD, the expression levels of EMT-related genes and transcription factors were investigated using western blotting and immunofluorescence assays. Following the transfection of PaTu8988 cells with si-FUBP1, western blotting revealed that the expression levels of E-cadherin were significantly upregulated, while the expression levels of N-cadherin, vimentin and $\beta$-catenin were downregulated (Fig. 5A). In contrast, the overexpression of FUBP1 in SW1990 cells demonstrated the opposite trend (Fig. 5B). Similarly, the results of the immunofluorescence assay revealed that the knockdown of FUBP1 expression upregulated E-cadherin 
A
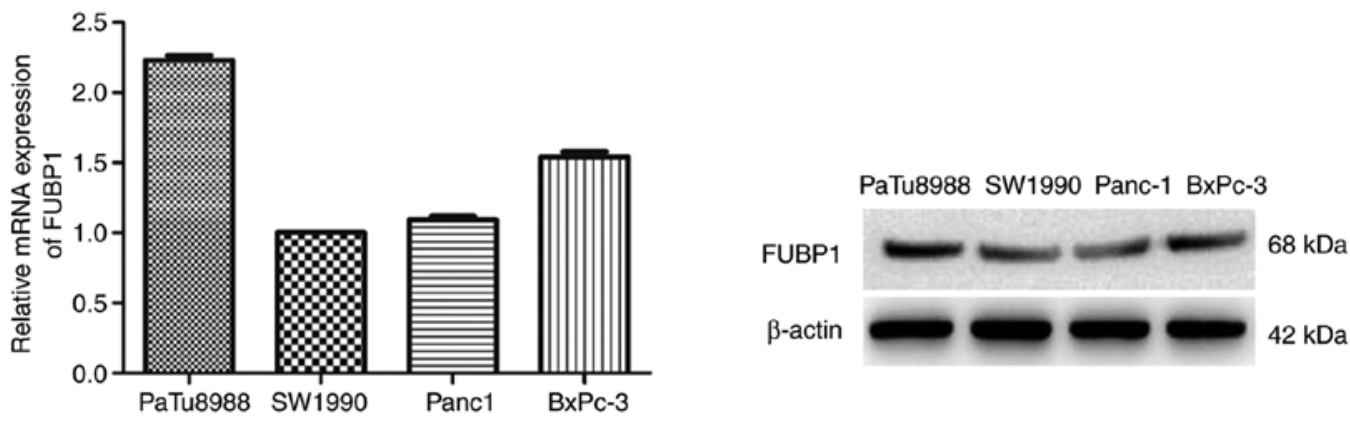

B
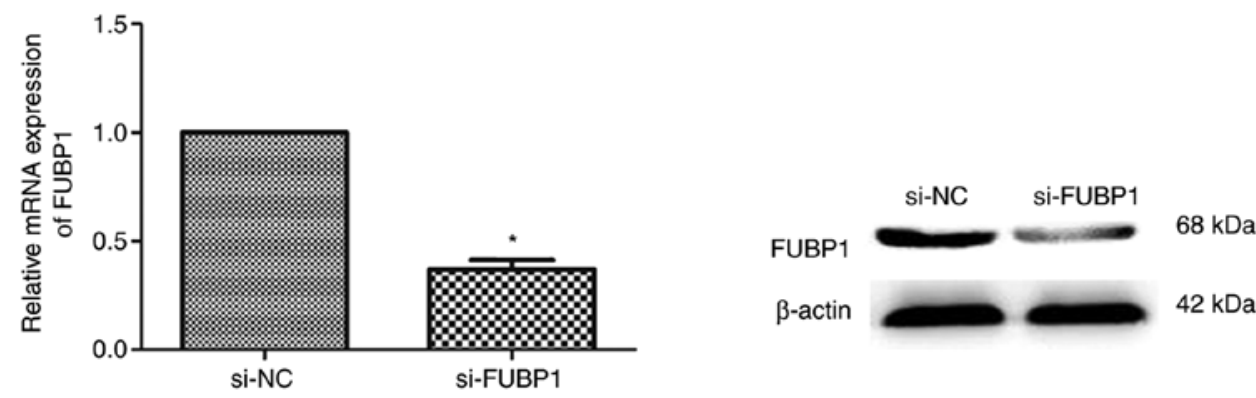

C
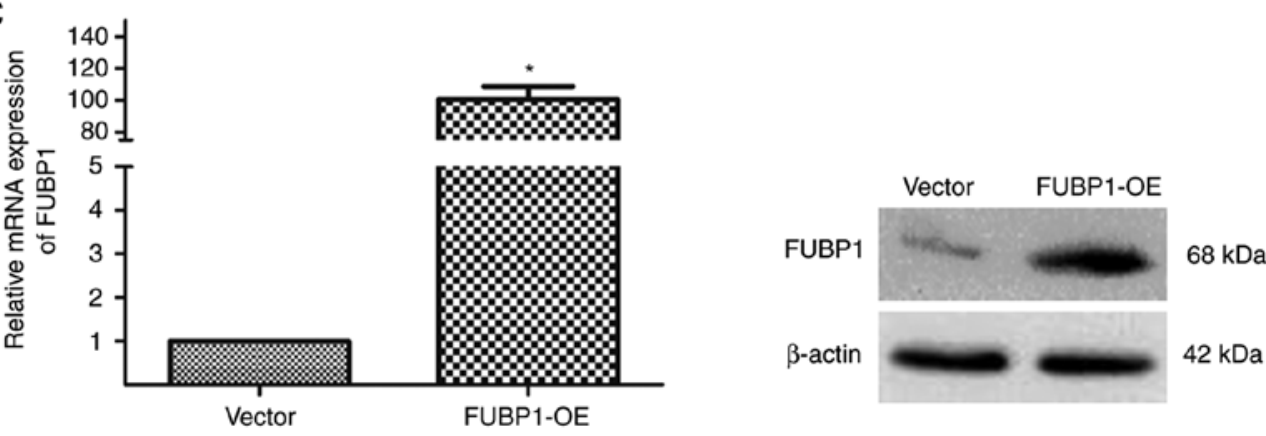

Figure 2. Expression levels of FUBP1 in PAAD cell lines. (A) mRNA and protein expression levels of FUBP1 in four human PAAD cell lines. (B) Transfection efficiency of si-FUBP1 or si-NC in PaTu8988 cells was analyzed using RT-qPCR and western blotting. (C) Transfection efficiency of FUBP1-OE or empty vector in SW1990 cells was analyzed using RT-qPCR and western blotting. Data are presented as the mean \pm SD from three independent experiments. $\mathrm{P}<0.05$. FUBP1, far upstream element-binding protein 1; PAAD, pancreatic adenocarcinoma; si, small interfering RNA; NC, negative control; OE, overexpression; RT-qPCR, reverse transcription-quantitative PCR.

A

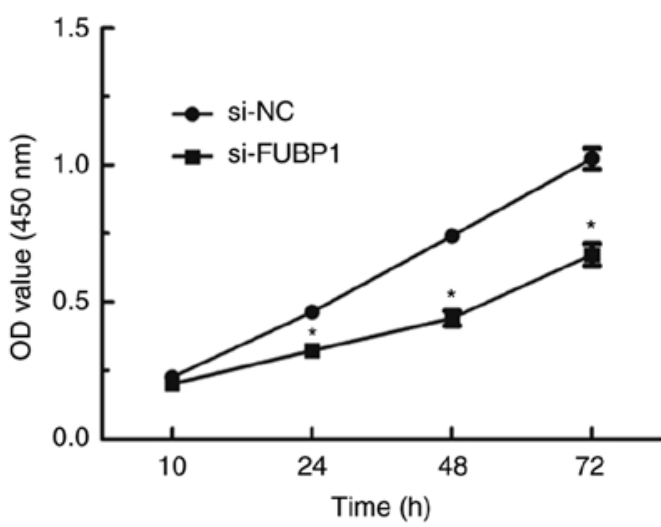

PaTu8988
B

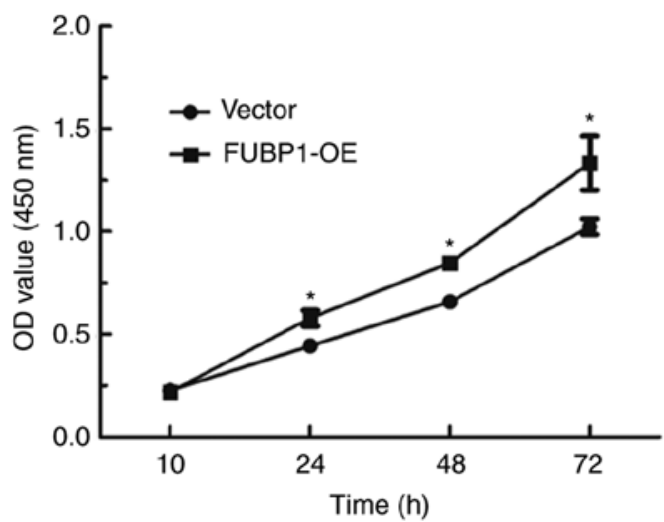

SW1990

Figure 3. FUBP1 promotes pancreatic adenocarcinoma cell viability. Viability of (A) PaTu8988 cells transfected with si-NC or si-FUBP1 and (B) SW1990 cells transfected with empty or FUBP1-OE vectors was analyzed using a Cell Counting Kit- 8 assay. Data are presented as the mean \pm SD from three independent experiments. " $\mathrm{P}<0.05$. FUBP1, far upstream element-binding protein 1; si, small interfering RNA; NC, negative control; OE, overexpression.

expression and downregulated vimentin expression (Fig. 5C). These observations suggested that FUBP1 may promote the transition of PAAD cells from an epithelial to mesenchymal phenotype. 
A
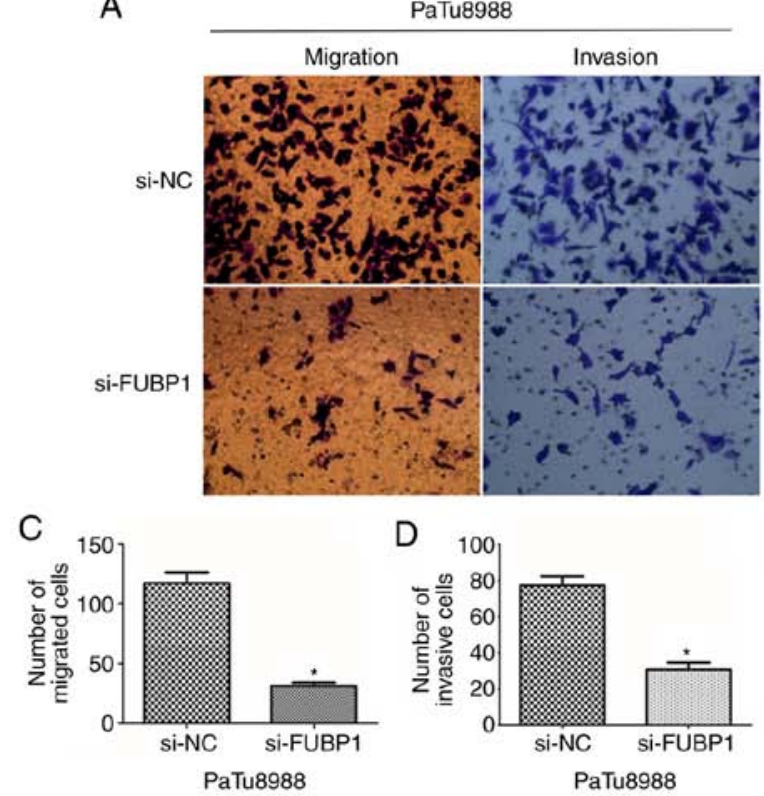

D 100

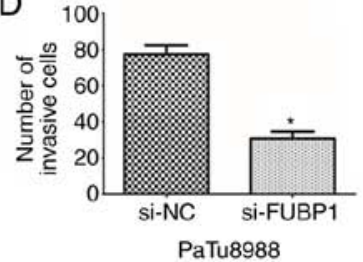

G
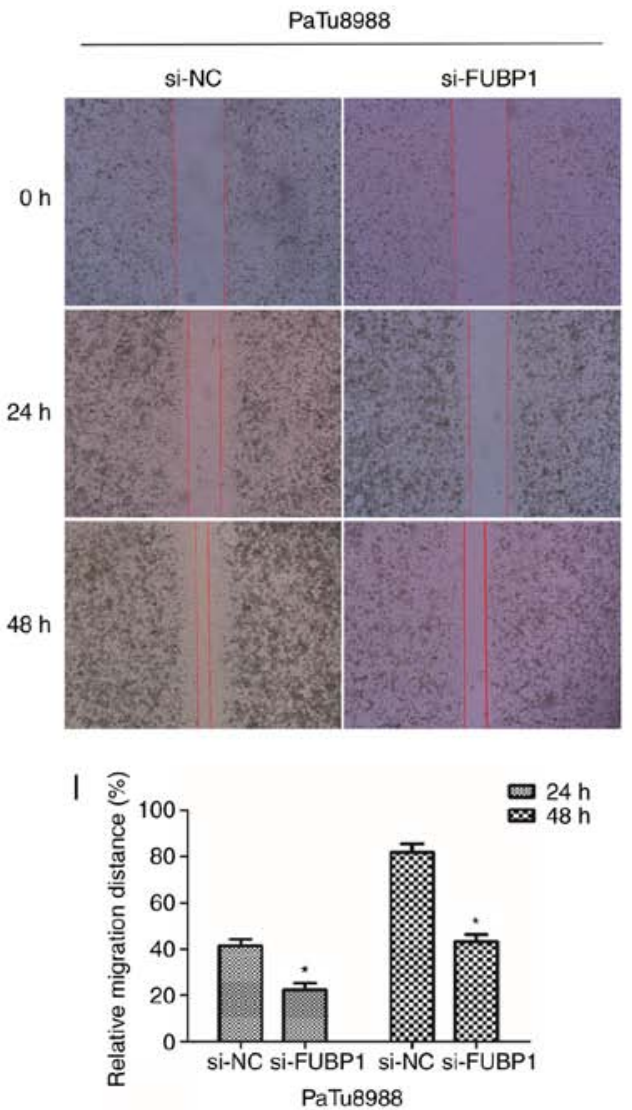

B

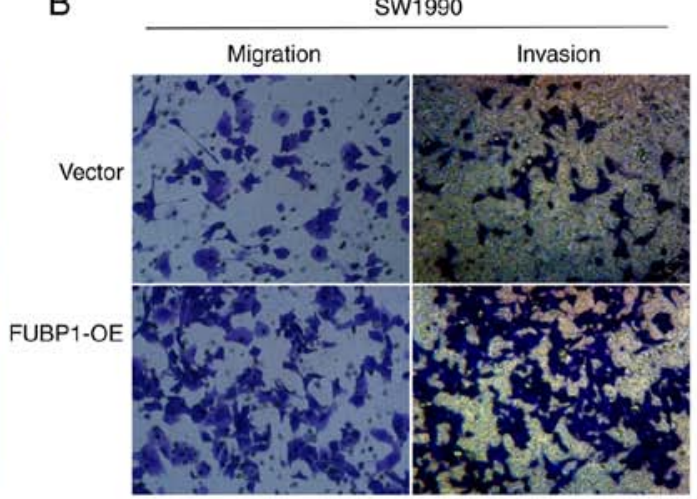

$\mathrm{E}$
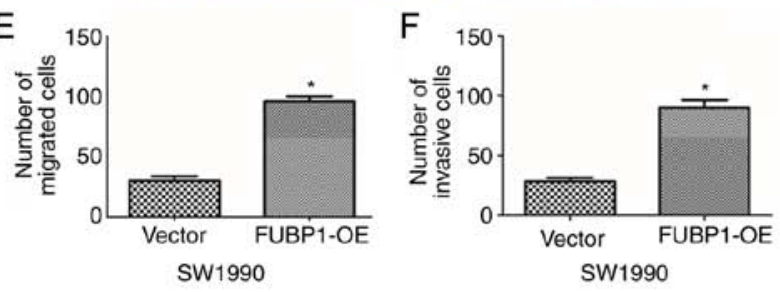

$\mathrm{H}$

SW1990

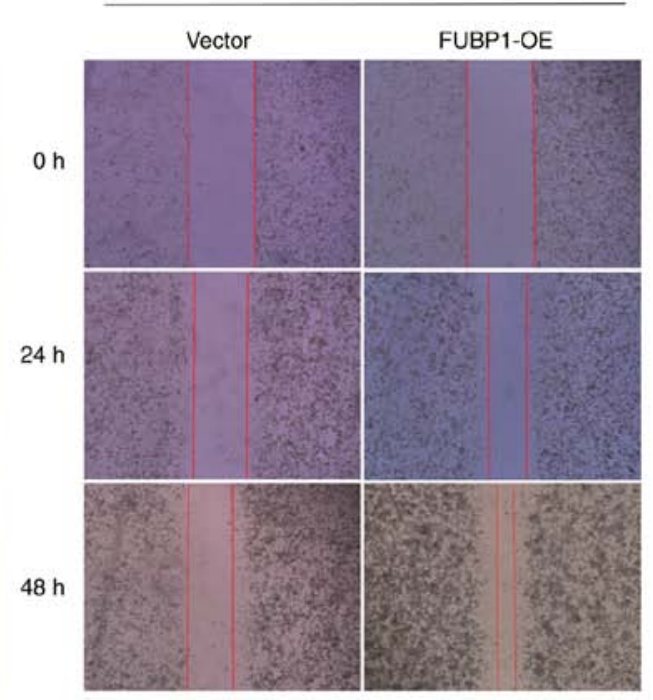

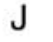

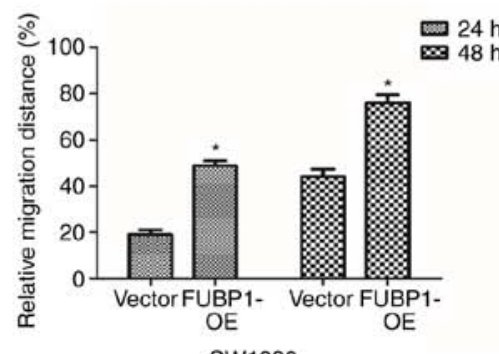

Figure 4. FUBP1 promotes pancreatic adenocarcinoma cell migration and invasion. Transwell assays were used to analyze the migratory and invasive abilities of (A) PaTu8988 cells transfected with si-NC or si-FUBP1 and (B) SW1990 cells transfected with FUBP1-OE or empty vectors (magnification, x200). (C and E) The number of migrated cells was counted and analyzed. (D and F) Number of invasive cells was counted and analyzed. Wound healing assays were used to determine the cell migratory ability of (G) PaTu8988 cells transfected with si-NC or si-FUBP1 and (H) SW1990 cells transfected with empty or FUBP1-OE vectors (magnification, x100). (I and J) The migratory distance was measured and analyzed. Data are presented as the mean \pm SD from three independent experiments. ${ }^{*} \mathrm{P}<0.05$. FUBP1, far upstream element-binding protein 1; si, small interfering RNA; NC, negative control; OE, overexpression.

Multiple previous studies have demonstrated that the TGF $\beta /$ Smad signaling pathway was a central regulator of cancer cell proliferation, metastasis and the EMT process $(22,25,26)$. Thus, the present study subsequently aimed to determine the molecular mechanisms through which FUBP1 exerted its functions. In a previous study, Kyoto Encyclopedia of Genes and Genomes signaling pathway enrichment analysis identified that FUBP1 was associated with the TGF $\beta$ signaling pathway, and FUBP1 promoted the EMT of hepatocellular carcinoma cells by activating the TGF $\beta /$ Smad signaling pathway (8). 


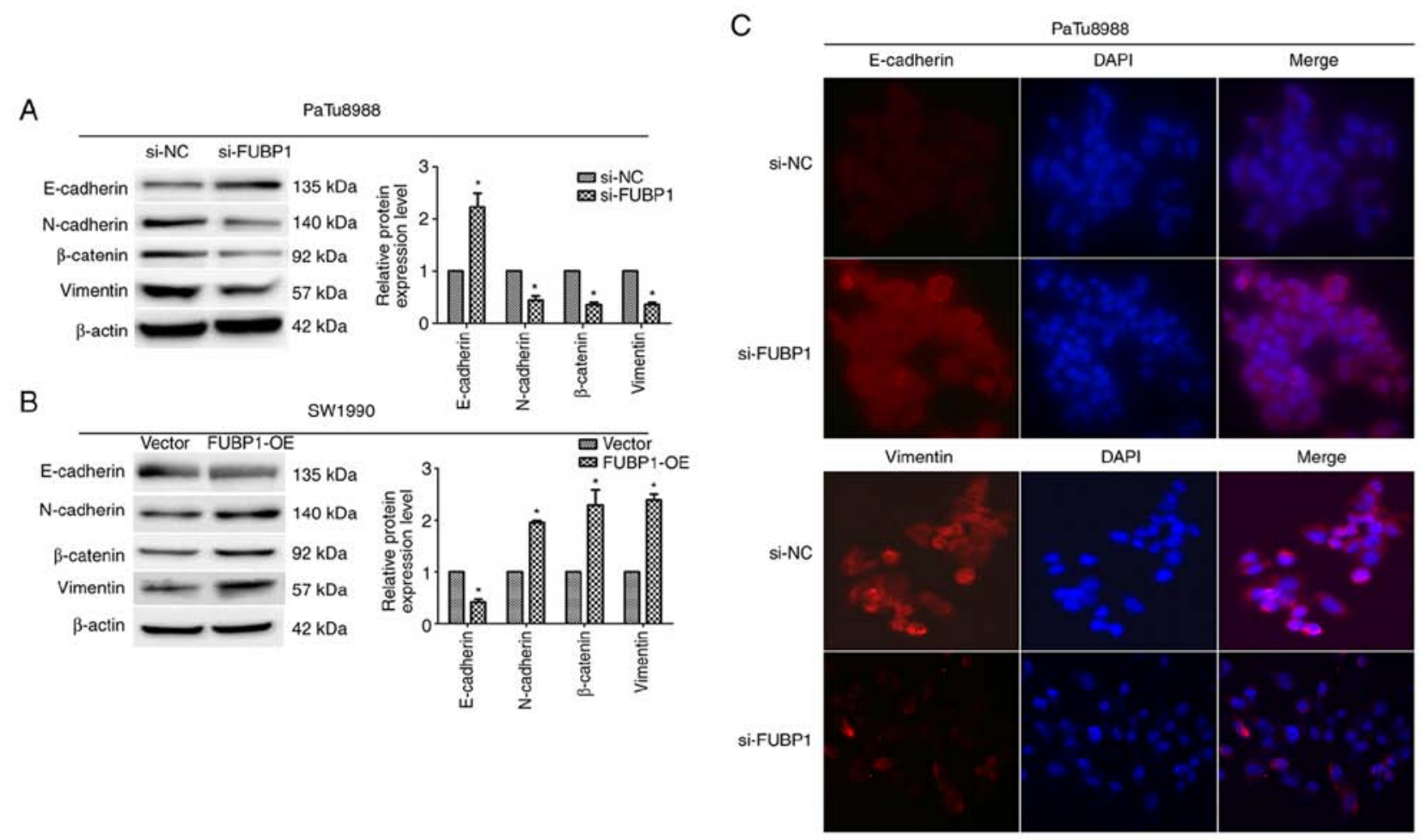

Figure 5. FUBP1 promotes EMT in pancreatic adenocarcinoma cells. Western blotting was used to analyze the expression levels of the EMT-related proteins, E-cadherin, N-cadherin, $\beta$-catenin and vimentin, in (A) PaTu8988 cells transfected with si-NC or si-FUBP1 and (B) SW1990 cells transfected with empty or FUBP1-OE vectors. $\beta$-actin was used as the internal loading control. (C) Immunofluorescence assay was used to determine E-cadherin and vimentin expression levels in PaTu8988 cells transfected with si-NC or si-FUBP1 (magnification, $x 200$ ). Data are presented as the mean \pm SD from three independent experiments. " $\mathrm{P}<0.05$. FUBP1, far upstream element-binding protein 1; EMT, epithelial-mesenchymal transition; si, small interfering RNA; NC, negative control; OE, overexpression.

A

PaTu8988

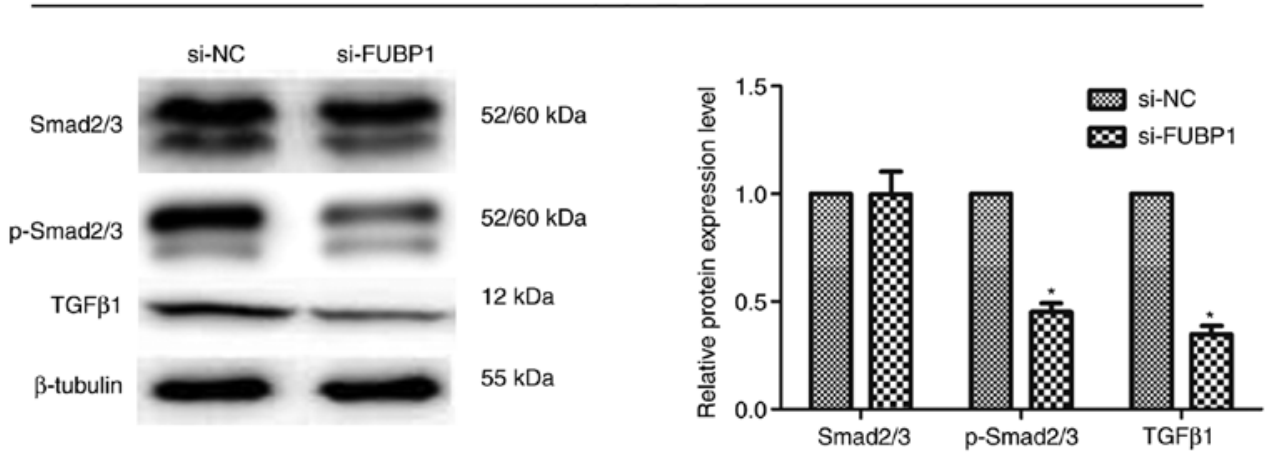

B

SW1990

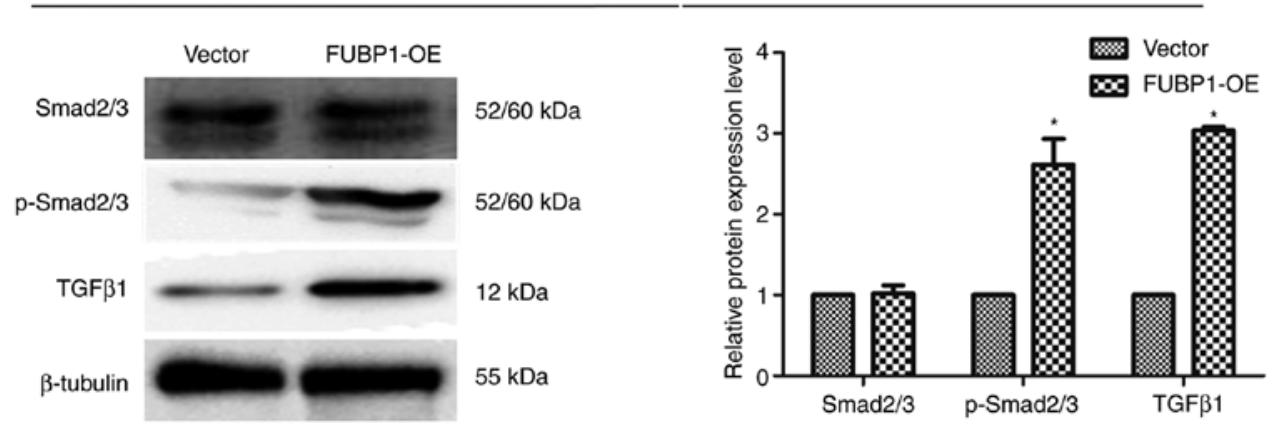

Figure 6. FUBP1 activates epithelial-mesenchymal transition via the TGF $/$ Smad signaling pathway in pancreatic adenocarcinoma cells. Western blotting was used to analyze the expression levels of the TGF $\beta /$ Smad signaling pathway proteins, Smad2/3, p-Smad2/3 and TGF $\beta 1$, in (A) PaTu8988 cells transfected with si-NC or si-FUBP1 and (B) SW1990 cells transfected with empty or FUBP1-OE vectors. $\beta$-tubulin was used as the internal loading control. Data are presented as the mean \pm SD from three independent experiments. ${ }^{*} \mathrm{P}<0.05$. FUBP1, far upstream element-binding protein 1; p-, phosphorylated; si, small interfering RNA; NC, negative control; OE, overexpression. 
Thus, whether FUBP1 could regulate EMT through the TGF $\beta /$ Smad signaling pathway in human PAAD cells was investigated. The expression levels of $\mathrm{p}-\mathrm{Smad} 2 / 3$ and TGF $\beta 1$, which are pivotal signaling molecules in the TGF $\beta / \mathrm{Smad}$ signaling pathway (27), were analyzed using western blotting. Notably, FUBP1 expression levels were found to be positively correlated with the expression levels of TGF $\beta /$ Smad signaling pathway target genes. The transfection of PaTu8988 cells with si-FUBP1 significantly downregulated $\mathrm{p}-\mathrm{Smad} 2 / 3$ and TGF $\beta 1$ expression levels (Fig. 6A), while the overexpression of FUBP1 in SW1990 cells significantly upregulated the expression levels of p-Smad2/3 and TGF $\beta 1$ (Fig. 6B). Collectively, these data indicated that FUBP1 may activate EMT through TGF $\beta /$ Smad signaling in human PAAD cells.

\section{Discussion}

The results of the present study revealed that the expression levels of FUBP1 were upregulated in human PAAD tissues compared with adjacent normal tissues in both clinical tissues and data from TCGA database. Survival analysis using the starBase database also demonstrated that patients with PAAD with upregulated FUBP1 expression levels were at a higher risk of PAAD-related mortality compared with patients with lower expression levels of FUBP1. These data suggested that FUBP1 expression levels may be upregulated in patients with PAAD and may be associated with a poor prognosis, which is consistent with the findings of Fan et al (18). The results of the CCK- 8 assay revealed that FUBP1 promoted cell proliferation. Moreover, wound healing and Transwell migration and invasion assays revealed that FUBP1 promoted PAAD cell migration and invasion in vitro. During EMT, epithelial cells transition from an epithelial to mesenchymal phenotype, which causes cells to lose their adhesive ability and acquire migratory and invasive properties. Characteristic changes during EMT include the downregulation of the expression levels of epithelial markers, such as E-cadherin, and the upregulation of mesenchymal markers, including N-cadherin, vimentin and EMT-related transcription factors, such as Snail, twist family bHLH transcription factor 1 and zinc finger E-box binding homeobox 1 (24). The results of the present study revealed that the knockdown of FUBP1 upregulated the expression levels of E-cadherin, downregulated vimentin, $\mathrm{N}$-cadherin and $\beta$-catenin expression levels, and reversed the progression of EMT. The TGF $\beta / \mathrm{Smad}$ signaling pathway is well established as a central regulator of cancer cell proliferation, metastasis and the EMT process (22-25). For example, a previous study demonstrated that FUBP1 promoted EMT by activating the TGF $\beta /$ Smad signaling pathway in hepatocellular carcinoma cells (8). However, to the best of our knowledge, the role of FUBP1 in TGF $\beta /$ Smad signaling pathway-mediated EMT has not been reported. Therefore, the present study hypothesized that FUBP1 may regulate the EMT process through the TGF $\beta /$ Smad signaling pathway. To address this hypothesis, the expression levels of proteins involved downstream of the TGF $\beta /$ Smad signaling pathway cascade were investigated in vitro. The results revealed that the knockdown of FUBP1 expression decreased p-Smad2/3 and TGF $\beta 1$ expression levels, while the overexpression of FUBP1 increased p-Smad2/3 and TGF $\beta 1$ expression levels.
These findings suggested that FUBP1 may activate EMT via the TGF $\beta /$ Smad signaling pathway in human PAAD cells in vitro.

In conclusion, the results of the present study revealed that FUBP1 expression levels were upregulated in patients with PAAD and the upregulated expression levels predicted a poor clinical prognosis. Moreover, the findings indicated that FUBP1 may promote the proliferation, invasion and metastasis of PAAD cells. To the best of our knowledge, the present study was the first to identify that FUBP1 served as a pivotal regulator of EMT in PAAD cells by regulating the TGF $\beta / \mathrm{Smad}$ signaling pathway. Thus, FUBP1 may serve as an oncogene that promotes PAAD cell proliferation and progression, and may serve as a clinically relevant prognostic biomarker or represent a novel therapeutic target for PAAD. Further preclinical studies and clinical trials will be required to determine whether FUBP1 can predict the benefit of prognosis in PAAD. In addition, further studies on tissues of PAAD patients are required to thoroughly understand the clinical features of FUBP1. In the future, the detailed mechanism of FUBP1, as well as the underlying effects of other genes regulated by FUBP1 in PAAD progression will be investigated.

\section{Acknowledgements}

Not applicable.

\section{Funding}

The present study was supported by Shanghai Municipal Health Commission Foundation (grant no. 20204Y0181) and Shanghai University of Medicine and Health Sciences Seed Foundation (grant no. SFP-18-22-15-002).

\section{Availability of data and materials}

All data generated or analyzed during this study are included in this published article.

\section{Authors' contributions}

$\mathrm{XZ}, \mathrm{HC}$ and JC conceived and designed the experiments and wrote the paper. $\mathrm{YZ}, \mathrm{XZ}$ participated in all the experiments. $\mathrm{HC}$ and JC contributed to the design of the study and interpretation of experimental results. NZ and YL performed and analyzed the experiments of western blotting. JL performed and analyzed the experiments of immunofluorescence. XX analyzed the data and revised this manuscript. All authors read and approved the final manuscript.

\section{Ethics approval and consent to participate}

All patients provided written informed consent, and the study was approved by the Ethics Committee of Shanghai Fengxian District Central Hospital (Shanghai, China).

\section{Patient consent for publication}

Not applicable. 


\section{Competing interests}

The authors declare that they have no competing interests.

\section{References}

1. Siegel RL, Miller KD and Jemal A: Cancer statistics, 2020. CA Cancer J Clin 70: 7-30, 2020.

2. Miller KD, Nogueira L, Mariotto AB, Rowland JH, Yabroff KR, Alfano CM, Jemal A, Kramer JL and Siegel RL: Cancer treatment and survivorship statistics, 2019. CA Cancer J Clin 69: 363-385, 2019.

3. Stornello C, Archibugi L, Stigliano S, Vanella G, Graglia B, Capalbo C, Nigri G and Capurso G: Diagnostic delay does not influence survival of pancreatic cancer patients. United European Gastroenterol J 8: 81-90, 2020.

4. Zhang J and Chen QM: Far upstream element binding protein 1: A commander of transcription, translation and beyond. Oncogene 32: 2907-2916, 2013.

5. Debaize L and Troadec MB: The master regulator FUBP1: Its emerging role in normal cell function and malignant development. Cell Mol Life Sci 76: 259-281, 2019.

6. Frost JR, Mendez M, Soriano AM, Crisostomo L, Olanubi O, Radko S and Pelka P: Adenovirus 5 E1A-Mediated Suppression of p53 via FUBP1. J Virol 92: e00439-18, 2018.

7. Rabenhorst U, Thalheimer FB, Gerlach K, Kijonka M, Böhm S, Krause DS, Vauti F, Arnold HH, Schroeder T, Schnütgen F, et al: Single-stranded DNA-binding transcriptional regulator FUBP1 is essential for fetal and adult hematopoietic stem cell self-renewal. Cell Rep 11: 1847-1855, 2015.

8. Fu PY, Hu B, Ma XL, Tang WG, Yang ZF, Sun HX, Yu MC, Huang A, Hu JW, Zhou CH, et al: Far upstream element-binding protein 1 facilitates hepatocellular carcinoma invasion and metastasis. Carcinogenesis 41: 950-960, 2020.

9. Baumgarten P, Harter PN, Tonjes M, Capper D, Blank AE, Sahm F von Deimling A, Kolluru V, Schwamb B, Rabenhorst U, et al: Loss of FUBP1 expression in gliomas predicts FUBP1 mutation and is associated with oligodendroglial differentiation, IDH1 mutation and $1 \mathrm{p} / 19 \mathrm{q}$ loss of heterozygosity. Neuropathol Appl Neurobiol 40: 205-216, 2014

10. Jiang P, Huang M, Qi W, Wang F, Yang T, Gao T, Luo C, Deng J, Yang Z,Zhou T, et al: FUBP1 promotes neuroblastoma proliferation via enhancing glycolysis-a new possible marker of malignancy for neuroblastoma. J Exp Clin Cancer Res 38: 400, 2019.

11. Duan J, Bao X, Ma X, Zhang Y, Ni D, Wang H, Zhang F, Du Q, Fan Y, Chen J, et al: Upregulation of far upstream element-binding protein 1 (FUBP1) promotes tumor proliferation and tumorigenesis of clear cell renal cell carcinoma. PLoS One 12: e0169852, 2017.

12. MullerB,BovetM,Yin Y,StichelD,MalzM,González-Vallinas M, Middleton A, Ehemann V, Schmitt J, Muley T, et al: Concomitant expression of far upstream element (FUSE) binding protein (FBP) interacting repressor (FIR) and its splice variants induce migration and invasion of non-small cell lung cancer (NSCLC) cells. J Pathol 237: 390-401, 2015.

13. Chen Y, Liu J, Geng N and Feng C: Upregulation of far upstream element-binding protein 1 (FUBP1) promotes tumor proliferation and unfavorable prognosis in tongue squamous cell carcinoma. Int J Biol Markers 35: 56-65, 2020.
14. Yang L, Zhu JY, Zhang JG, Bao BJ, Guan CQ, Yang XJ, Liu YH, Huang YJ, Ni RZ and Ji LL: Far upstream element-binding protein 1 (FUBP1) is a potential c-Myc regulator in esophageal squamous cell carcinoma (ESCC) and its expression promotes ESCC progression. Tumour Biol 37: 4115-4126, 2016.

15. Venturutti L, Cordo Russo RI, Rivas MA, Mercogliano MF, Izzo F, Oakley RH, Pereyra MG, De Martino M, Proietti CJ, Yankilevich P, et al: MiR-16 mediates trastuzumab and lapatinib response in ErbB-2-positive breast and gastric cancer via its novel targets CCNJ and FUBP1. Oncogene 35: 6189-6202, 2016.

16. Zhang F, Tian Q and Wang Y: Far upstream element-binding protein 1 (FUBP1) is overexpressed in human gastric cancer tissue compared to non-cancerous tissue. Onkologie 36: 650-655, 2013.

17. Jia MY and Wang YJ: Far upstream element-binding protein 1 (FUBP1) expression differs between human colorectal cancer and non-cancerous tissue. Neoplasma 61: 533-540, 2014.

18. Fan P, Ma J and Jin X: Far upstream element-binding protein 1 is up-regulated in pancreatic cancer and modulates immune response by increasing programmed death ligand 1 . Biochem Biophys Res Commun 505: 830-836, 2018.

19. Livak KJ and Schmittgen TD: Analysis of relative gene expression data using real-time quantitative PCR and the 2(-Delta Delta C(T)) method. Methods 25: 402-408, 2001

20. Goldman MJ, Craft B, Hastie M, Repečka K, McDade F, Kamath A, Banerjee A, Luo Y, Rogers D, Brooks AN, et al: Visualizing and interpreting cancer genomics data via the Xena platform. Nat Biotechnol 38: 675-678, 2020.

21. Li JH, Liu S, Zhou H, Qu LH and Yang JH: StarBase v2.0: Decoding miRNA-ceRNA, miRNA-ncRNA and protein-RNA interaction networks from large-scale CLIP-Seq data. Nucleic Acids Res 42: D92-97, 2014.

22. van Staalduinen J, Baker D, Ten Dijke P and van Dam H: Epithelial-mesenchymal-transition-inducing transcription factors: New targets for tackling chemoresistance in cancer? Oncogene 37: 6195-6211, 2018.

23. Lamouille S, Xu J and Derynck R: Molecular mechanisms of epithelial-mesenchymal transition. Nat Rev Mol Cell Biol 15: 178-196, 2014.

24. Zhang X, Feng W, Zhang J, Ge L, Zhang Y, Jiang X, Peng W, Wang D, Gong A and Xu M: Long noncoding RNA PVT1 promotes epithelialmesenchymal transition via the TGF $\beta / S m a d$ pathway in pancreatic cancer cells. Oncol Rep 40: 1093-1102, 2018.

25. Zhao L, Liu S, Che X, Hou K, Ma Y, Li C, Wen T, Fan Y, Hu X, Liu Y and Qu X: Bufalin inhibits TGF- $\beta$-induced epithelial-to-mesenchymal transition and migration in human lung cancer A549 cells by downregulating TGF- $\beta$ receptors. Int J Mol Med 36: 645-652, 2015.

26. Park JH, Yoon J, Lee KY and Park B: Effects of geniposide on hepatocytes undergoing epithelial-mesenchymal transition in hepatic fibrosis by targeting TGF $\beta /$ Smad and ERK-MAPK signaling pathways. Biochimie 113: 26-34, 2015.

27. Valcourt U, Kowanetz M, Niimi H, Heldin CH and Moustakas A TGF-beta and the Smad signaling pathway support transcriptomic reprogramming during epithelial-mesenchymal cell transition. Mol Biol Cell 16: 1987-2002, 2005.

This work is licensed under a Creative Commons Attribution-NonCommercial-NoDerivatives 4.0 International (CC BY-NC-ND 4.0) License. 